JRDMS Journal of Research in Dental and Maxillofacial Sciences

\title{
The Effects of Diode Laser as an Adjunct to Scaling and Root Planing on Treatment of Chronic Periodontitis: A Review of the literature
}

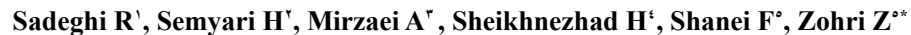 \\ ${ }^{l}$ Assistant Professor, Periodontology Dept, Faculty of Dentistry, Shahed University, Tehran, Iran \\ ${ }^{2}$ Associate Professor, Periodontology Dept, Faculty of Dentistry, Shahed University, Tehran, Iran. \\ ${ }^{3}$ AALZ,Aachen, Germany \\ ${ }^{4}$ Assistant Professor, Prosthodontics Dept, School of Dentistry, Guilan University, Rasht, Iran. \\ ${ }^{5}$ Post-Graduate Student, Periodontology Dept, Faculty of Dentistry, Shahed University, Tehran, Iran
}

\begin{tabular}{l}
\hline ARTICLE INFO \\
\hline Original Article \\
Article History \\
Received: Jan 2017 \\
Accepted:Feb 2017 \\
ePublished: Apr 2017 \\
\hline
\end{tabular}

Keywords:

Chronic periodontitis,

Laser,

Microbiology,

Treatment

\section{ABSTRACT}

\begin{abstract}
Background and aim: The most common form of periodontitis is chronic periodontitis, which is a destructive inflammatory disease of periodontal tissues and is usually associated with pocket formation, changes in density and height of alveolar bone, and sometimes gingival recession. Some patients are resistant to periodontitis treatment due to a weak immune system, smoking, and sometimes due to unknown reasons. Moreover, surgery is impossible in some cases; therefore, clinicians have to approach alternative methods such as laser therapy to achieve successful treatment outcomes. Since there are great differences among the results of previous clinical trials, a review study to investigate the materials and methods section of these studies seems necessary in order to find the reason of the controversies. The methodology of all the intervention studies that have evaluated the effect of diode laser on periodontitis treatment from 1997 to 2017 were examined and the results were reported.

Conclusion: randomized controlled trials should comply with the correct protocol. All the details of the treatment protocol and severity of periodontitis should be recorded in order to achieve reliable results. It can be concluded that the results of some of the reviewed studies are not reliable.
\end{abstract}




\section{Introduction:}

Periodontitis is a destructive inflammatory disease of periodontal tissues that is usually associated with pocket formation, changes in density and height of alveolar bone, and sometimes marginal gingival recession without pocket formation. Periodontitis is divided into three main categories based on the clinical, radiographic and laboratory findings and the patient's history. It includes chronic periodontitis, aggressive periodontitis and periodontitis as a manifestation of systemic disease. ${ }^{(1)}$

Chronic periodontitis is the most common manifestation, which is considered as an inflammatory disease with a slow progression. However, environmental and systemic factors may change the response to the biofilms of the host immune system and therefore, the disease will find a progressive destructive path. ${ }^{(2)}$ Treatment of periodontal disease is divided into two general categories: surgical treatment and non-surgical treatment that involves the use of various instruments in dentogingival complex..$^{(3)}$

During the initial course of periodontal therapy, root surfaces are debrided via scaling and root planing (SRP) with hand instruments or by power-driven instruments. ${ }^{(4)}$ In periodontal pockets, different strategies contribute to bacterial survival such as swimming of bacteria in gingival crevicular fluid (GCF), invading the pocket epithelium, and colonization of bacteria within dentinal tubules when the dentin is exposed. ${ }^{(5)}$ It is obvious that complete removal of bacterial deposits and toxins from root surfaces and periodontal pocket is not always feasible with the use of conventional mechanical methods. ${ }^{(4)}$ In addition, access to furcation area, concavities, grooves and distal surfaces of molars is limited. ${ }^{(5)}$

Systemic antibiotics can be used as an adjunct therapy and are useful for treatment of aggressive periodontitis and progressive chronic periodontitis. Local areas that do not respond to treatment, or sites where the disease recurs frequently can be treated with topical antibiotics. Since high concentrations of antibiotics are necessary for such purposes and some cases of resistance against antibiotics have been detected ${ }^{(6)}$, clini- cians felt the need for adjunct treatment methods such as laser therapy. ${ }^{(7-9)}$

Some patients are resistant to periodontitis treatment due to a weak immune system, smoking habits, and sometimes due to unknown reasons. Moreover, surgery is impossible in some cases; therefore, clinicians have to approach alternative therapies to achieve successful treatment outcomes. These techniques include ENAP, use of local and systemic antibiotics, photodynamic therapy and laser therapy with various success rates. In the studies of nearly three decades, a special attention has been paid to the application of lasers in dentistry. ${ }^{(10)}$

Laser has excellent tissue ablation ability with a strong bactericidal effect. It is one of the most promising new technical modalities for non-surgical periodontal treatment. Another advantage of laser treatment is the ability to access areas that are impossible to reach with conventional mechanical treatment. Laser irradiation has bactericidal effect while it does not create a smear layer, so that the surface of the treated root can become favorable for attachment of periodontal tissue. Conventionally, gingival curettage has been used for soft tissue debridement. Nevertheless, some studies have shown that gingival curettage after SRP with hand instruments has no added advantage over conventional SRP, although poor results of gingival curettage may be due to the absence of efficient instruments for soft tissue debridement. Proper removal of the epithelial lining of the pocket's soft tissue wall with laser can improve periodontal tissue regeneration. Part of the laser energy will turn into scattered radiation, which can irritate surrounding tissue cells, reduce inflammation and cell proliferation, and increase the lymph flow. It can improve periodontal tissue attachment and possibly reduce postoperative pain. ${ }^{(4)}$ Semiconductor diode lasers are available in three wavelengths of 810-830 nm, $940 \mathrm{~nm}$ and $980 \mathrm{~nm} .{ }^{(11)}$ Studies have shown that these wavelengths have a greater impact on orange and red bacteria associated with periodontitis because of strong absorption by pigments. ${ }^{(12)}$

Moritz et al reported that the bleeding index will be improved in $96.9 \%$ of patients under- 
going periodontal treatment assisted with laser therapy, while this value is equal to $66.7 \%$ in patients who are treated with conventional methods. This study shows that periodontal treatment with diode laser induces an antibacterial effect and reduces inflammation; therefore, it helps heal periodontal pockets by eliminating the bacteria. ${ }^{(13)}$

There are few contraindications for laser treatment with antibacterial effect. There are no concerns about bacterial resistance or allergic reactions. Lasers can be used in children, pregnant women, immuno-compromised patients and patients with pacemakers, defibrillators or other medical devices in the body. ${ }^{(11)}$

Since there are great differences among the results of previous clinical trials, a review study to investigate the materials and methods section of these studies seems necessary in order to find the reason of the controversies. The aim of the present review study is to investigate the recent intervention studies related to the performance of diode laser therapy as an adjunct to non-surgical periodontal therapy in treatment of chronic periodontitis in adults. The methodology of all the intervention studies that have evaluated the effect of diode laser on periodontitis treatment from 1997 to 2017 were examined and the results were reported. We did not exclude any randomized clinical trials.

\section{Clinical trials conducted to evaluate the effect of diode laser on treatment of chronic peri- odontitis}

Despite the widespread use of diode lasers in treatment of mild to moderate periodontitis, it is interesting to know that to date only 13 intervention studies have been published, in which diode laser treatment has been considered as an adjunct treatment to SRP and has been compared with conventional scaling. All the features of these studies including test and control groups, details of laser therapy (power, mode of radiation, and diameter of probe and wavelength), study duration and number of subjects are presented in Table 1.
Seven studies have used the wavelength between 805 and $830 \mathrm{~nm}:^{(13-19)}$

In 1998, Moritz et al performed a study on 50 patients. Bacterial reduction was more significant in test group after 6 months. Bleeding on probing (BOP) reduced by $96.9 \%$ in test group and by $66.7 \%$ in control group. Pocket depth significantly decreased in test group. ${ }^{(13)}$ In 2005 , Kreisler et al assessed the clinical effectiveness of laser on 22 patients (492 teeth) 3 months after treatment using split mouth method. A significant reduction in mobility, clinical attachment loss (CAL), and pocket depth (PD) was seen in the group which had received laser treatment in comparison with the control group. The two groups showed no significant differences in terms of periodontal index $(\mathrm{PI})$, gingival index (GI), bleeding on probing (BOP), and sulcus fluid flow rate (SFFR). According to the findings, it was concluded that the improved treatment in test group was mostly due to improved connective tissue attachment following laser deepithelialization, rather than the reduction of pathogens in periodontal pockets. ${ }^{(14)}$ In 2014, Üstün et al used the same method on 22 patients with generalized chronic periodontitis, and clinical and biochemical factors were recorded and compared at 1, 3 and 6 months after treatment. Only single-rooted teeth were investigated. The authors stated that during the 6 months of study, the healing and periodontal variables and IL1B levels were more satisfactory in test group compared with control group. ${ }^{(15)}$ In the study by Euzebio Alves et al in 2013, laser irradiation at $808 \mathrm{~nm}$ wavelength was performed in two stages one week apart. All clinical parameters (BOP, $\mathrm{PD}, \mathrm{CAL}$ ) in both groups were improved. But there was no statistically significant difference between the two groups. Also, microbial analysis showed a significant bacterial reduction in the two groups (especially black-pigmented bacteria), although the difference was not significant between test and control groups. ${ }^{(16)}$ In 1997, Moritz et al reported a significant reduction of microbial load, in particular Aggregatibacter actinomycetemcomitans (Aa), in subgingival pockets with depth of at least $4 \mathrm{~mm} .{ }^{(17)}$ In 2011, De Micheli et al reviewed the effectiveness of $808 \mathrm{~nm}$ diode 
laser and concluded that in laser group, a significant reduction was seen in PD and CAL; however, BOP in both groups showed similar results. In terms of microbiological parameters, no significant difference was observed between the two groups. (18) Jose et al in 2016 conducted a study on 15 patients to evaluate the effects of diode laser and topical application of Chlorhexidine (CHX) as adjunctive periodontal treatments. After 3 months, $\mathrm{CHX}+\mathrm{SRP}$ group and $\mathrm{CHX}+$ laser+SRP group showed the best results in terms of reduced PD and CAL. The difference was statistically significant. However, laser+SRP group did not show a statistically significant difference with control group. ${ }^{(19)}$

Four studies have used diode laser with the wavelength of $980 \mathrm{~nm}::^{(5,20-22)}$

In 2008, Caruso et al reviewed the effect of $980 \mathrm{~nm}$ diode laser on 13 patients with chronic periodontitis. Changes of 8 pathogens before treatment and one month after treatment were analyzed by PCR techniques. The clinical effect of diode laser as an adjunct therapy after 1,2 and 3 months was reported as remarkable, while the improvement has been more significant in BOP. No significant difference was detected between the two groups in terms of reduction in bacterial count. ${ }^{(5)} \mathrm{In}$ 2014, Zare et al reported that except for BOP which showed a significant reduction in laser group, other parameters were similar in both groups. The researchers have explained that laser irradiation may reduce inflammation in the inner layer of the pocket which leads to BOP reduction; however, laser has no effect on the external layer of the pocket (marginal inflammatory symptoms). Therefore, the results of modified gingival index (MGI) showed no significant difference between the two groups. ${ }^{(20)}$ In 2013, Dukić et al conducted a research with split mouth method on 35 patients with chronic periodontitis. Laser treatment was performed at 1, 3 and 7 days after SRP. Laser treatment caused a significant reduction in pocket depth only in pockets with depth of 4-6 mm. ${ }^{(21)}$ Borrajo et al examined 30 patients with mod- erate periodontitis. After 6 weeks, PBI was 0.24 in test group and 0.43 in control group. BOP reduction in control group was $19.5 \%$ less than that in test group. Nonetheless, CAL showed no significant difference between the two groups. ${ }^{(22)}$

Only two studies have used $940 \mathrm{~nm}$ diode laser: ${ }^{23,24)}$

In 2014, Saglam et al evaluated the efficacy of $940 \mathrm{~nm}$ diode laser as adjunct therapy for chronic periodontitis in 30 patients. In comparison of clinical parameters (CAL, PD, BOP, and GI), laser group showed better treatment results. In both groups, IL1B, IL6, MMP1 (Matrix metalloproteinase), MMP8, and TIMP1 (Tissue inhibitor of metalloproteinase) reduced after treatment while IL8 increased. In laser group, IL8 level showed a more significant increase than control group in first month. ${ }^{(23)}$ In 2015, Nguyen et al studied 22 patients who had been treated with SRP and were in the maintenance course, in order to evaluate the effectiveness of diode laser with $0.8 \mathrm{~W}$ power in treatment of chronic periodontitis. PD, CAL and BOP clinical parameters and inflammatory factor IL1B were compared 3 months after treatment, and no statistically significant difference was detected between the two groups. ${ }^{(24)}$

Of the mentioned studies, only five studies have conducted microbial tests in order to examine the laser effects on pathogens. ${ }^{(5,13,16-18)}$

Of course, the methods of these studies need to be discussed. It is obvious that Meta-analysis studies may fail due to inadequate number of studies and variations in the design of therapeutic interventions, and also due to unreliable results of intervention studies caused by applying inappropriate treatment protocols. 


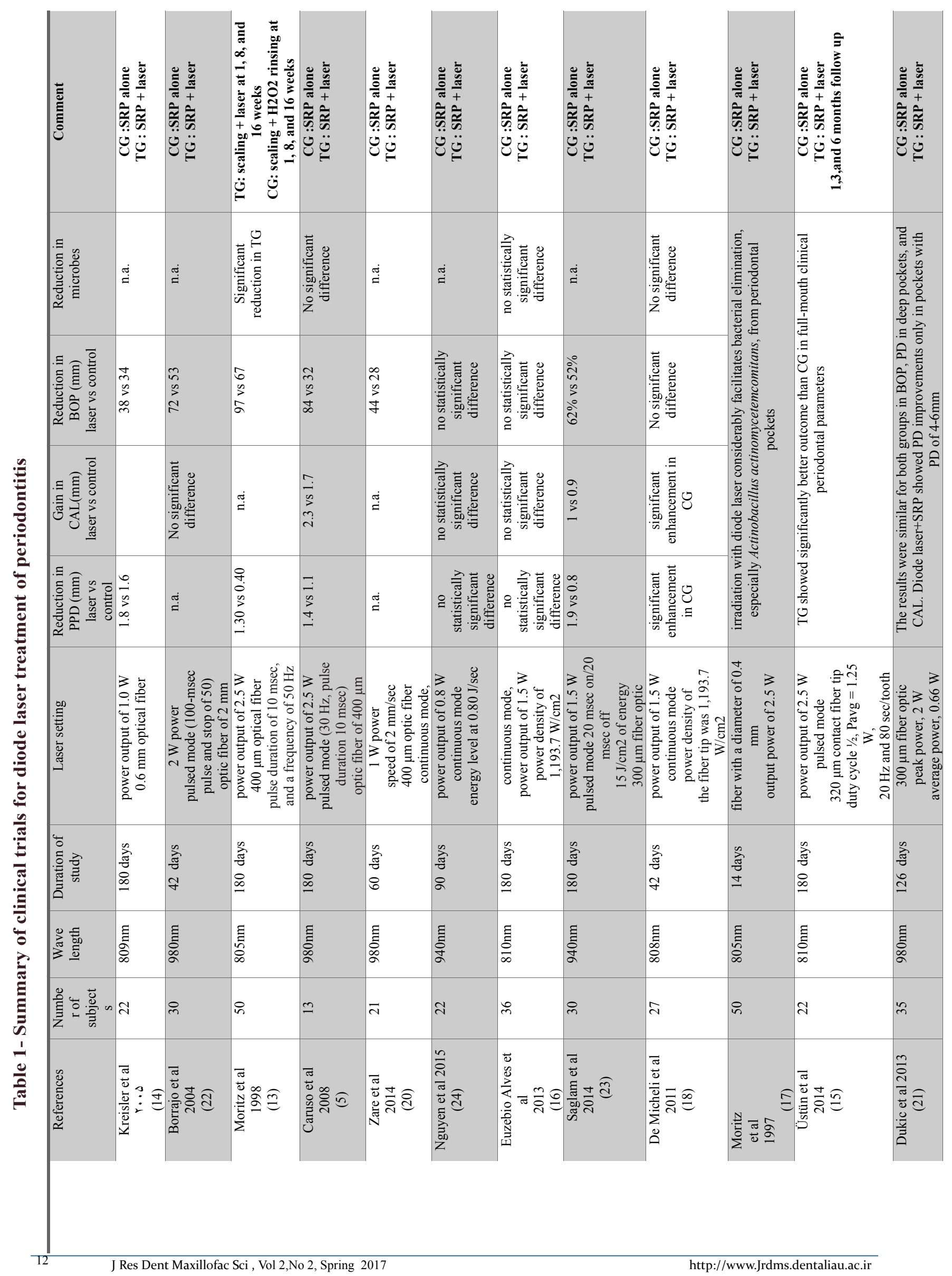




\section{Discussion:}

Since each of these studies has investigated some of the clinical parameters and has reported different results, we decided to conduct a detailed review of PPD, CAL, and MGI clinical parameters and microbial analyses, and to compare their research methods through reviewing intervention studies that have assessed the efficiency of diode laser in chronic periodontitis treatment.

In regards to pocket depth, Euzebio Alves et al used $808 \mathrm{~nm}$ diode laser on single-rooted teeth, and observed no significant difference between the two study groups. It should be considered that Euzebio Alves and colleagues examined single-rooted teeth which usually show good results after conventional treatments. Consequently, this result is not unexpected. ${ }^{(16)}$ Jose et al conducted a study using $810 \mathrm{~nm}$ diode laser, and did not report a significant effect on PD. It should be considered that the authors have used the power of $0.5 \mathrm{~W}$ for 10 seconds, which may not be adequate. ${ }^{(19)}$ Nguyen et al assessed the effect of $940 \mathrm{~nm}$ diode laser and found no significant effect on PD compared to treatment with SRP alone. It should be noted that in this study, laser irradiation and SRP were performed simultaneously, and that power of $0.8 \mathrm{~W}$ may be insufficient for therapeutic purposes. ${ }^{(24)}$

In regards to CAL, De Micheli et al reported the significant impact of $808 \mathrm{~nm}$ diode laser on reducing CAL compared with control group; which can be due to application of a different protocol in terms of the times of laser irradiation ( 1 day and 1 week after completion of scaling) or use of a different wavelength. (18) Also, Üstün et al stated that the use of $810 \mathrm{~nm}$ diode laser as an adjunct to SRP produced significant improvements in CAL. ${ }^{(15)}$

In a study about gingivitis, Jose et al did not observe a significant difference between laser group irritated with the wavelength of $810 \mathrm{~nm}$ and control group in terms of decreasing GI, which can be caused by irradiation settings (power of $0.5 \mathrm{~W}$ for 10 seconds). ${ }^{(19)}$ Previous studies have shown that laser radiation can affect the production of cytokines and growth factors, and thereby can accelerate the recovery. ${ }^{(3,6,25)}$ Üstün et al showed that the use of $810 \mathrm{~nm}$ diode laser can cause significant decrease in the amount of GCF and IL-1 $\beta$ compared to control group, which could be an explanation for the decrease in GI and PD. ${ }^{(15)}$

In microbial analysis, PCR method was used in the research by Caruso et al which can only detect the presence or absence of bacteria with no ability to detect small bacterial changes. ${ }^{(5)}$
In studies by De Micheli et al and Euzebio Alves et al, microbial culture method was used; however, it is very difficult to culture anaerobic bacteria and this may have influenced the conclusions. ${ }^{(16,18)}$

Since the number of studies that have conducted microbial analyses is limited, the differences in treatment protocols and methodologies used for microbial analysis or in examination of the pathogens have become problematic. However, the review of the microbial studies shows that the majority of these studies agree on the reduction of pathogens. Some of them have compared 805 $\mathrm{nm}$ diode laser, as an adjunct therapy for periodontitis, with control group and have found a more significant reduction in Prevotella Intermedia (PI) and Porphyromonas Gingivalis (PG) in laser group. In separate studies, Zare et al and Caruso et al assessed $980 \mathrm{~nm}$ diode laser; however, Caruso et al did not observe a significant difference after six months in contrast to the findings of Zare et al. ${ }^{(5,26)}$ It is noteworthy that they used different biological analysis methods. Caruso et al used PCR method but only descriptively reported those samples that were free from pathogens, without performing statistical analysis on changes of periodontal pathogens, since basically PCR test lacks comparability for the number of pathogens. ${ }^{(5)}$

Euzebio Alves et al and De Micheli et al selected $808 \mathrm{~nm}$ diode laser as an adjunct therapy and examined the changes of PG and PI pathogens in microbial culture. They reported that the laser did not cause a significant pathogen reduction compared with conventional treatment methods. ${ }^{(16,18)}$ It is noteworthy that in the research by Euzebio Alves et al, samples under examination were single-rooted teeth which due to their morphology and adequate access, they usually respond well to conventional therapies. Therefore, the results were expectable.

The first possible reason for the insignificant impact of laser in some studies or for the difference among the results of the studies that have worked on the same laser wavelength can be attributed to non-compliance with the correct protocol during laser treatment including the use of proper and effective laser power against pathogens which is yet safe for the periodontium, cleaning thefiberhead during work to avoid the lossofoutput 
power, proper irradiation duration with respect to the surface of the tooth, and laser irradiation when there is minimum blood in the pocket, since blood can prevent the laser from reaching the pathogens.

Finally, in order to avoid bias, we are obliged to exclude those subjects who are resistant to conventional therapy due to systemic diseases, consumption of certain drugs or smoking habits and therefore, the samples are expected to respond well to conventional treatments. Consequently, the difference with the results of laser group may not be significant and the use of laser may become logically unjustifiable, especially in studies that have only investigated single-rooted teeth. Further studies on patients who have not responded to SRP are recommended so that the effect of laser, as a potential adjunct therapy, can be more thoroughly investigated.

\section{Conclusion}

Randomized controlled trials should comply with the correct protocol (energy, time, mode, capacity and type of laser). All the details of the treatment protocol and severity of periodontitis should be recorded in order to achieve reliable results. It can be concluded that the results of some of the reviewed studies are not reliable.

Diode lasers can promote periodontal health and can be a supplement to an accurate root debridement. Supplemented periodontal treatments lead to a significant reduction of periopathogens and thereby help maintain periodontal health. Understanding the application and safety of laser treatment methods provides higher treatment standards.

\section{References:}

1. Smiley CJ, Tracy SL, Abt E, Michalowicz BS, John MT, Gunsolley J, Cobb CM, Rossmann J, Harrel SK, Forrest JL, Hujoel PP. Systematic review and meta-analysis on the nonsurgical treatment of chronic periodontitis by means of scaling and root planing with or without adjuncts. J Am Dent Assoc. 2015 Jul 31;146(7):508-24

2. Flemmig TF. Periodontitis. Ann Periodontol. 1999;4(1):32-7.
3. Badersten A, Nilvéus R, Egelberg J. Effect of nonsurgical periodontal therapy. J Clin Periodontol. $1981 ; 8(1): 57-72$.

4. Aoki A, Sasaki KM, Watanabe H, Ishikawa I. Lasers in nonsurgical periodontal therapy. Periodontol 2000. 2004;36(1):59-97.

5. Caruso U, Nastri L, Piccolomini R, d'Ercole S, Mazza C, Guida L. Use of diode laser $980 \mathrm{~nm}$ as adjunctive therapy in the treatment of chronic periodontitis. A randomized controlled clinical trial. New Microbiol. 2008;31(4):513-8.

6. Chambrone L, Vargas M, Arboleda S, Serna M, Guerrero M, de Sousa J, Lafaurie GI. Efficacy of Local and Systemic Antimicrobials in the NonSurgical Treatment of Smokers With Chronic Periodontitis: A Systematic Review. J Clin Periodontol.2016 Nov;87(11):1320-32.

7. Mullins SL, MacNeill SR, Rapley JW, Williams KB, Eick JD, Cobb CM. Subgingival microbiologic effects of one-time irradiation by $\mathrm{CO} 2$ laser: A pilot study. J Periodontol. 2007;78(12):2331-7. 8. Barone A, Covani U, Crespi R, Romanos G. Root surface morphological changes after focused versus defocused $\mathrm{CO} 2$ laser irradiation: a scanning electron microscopy analysis. J Periodontol. 2002;73(4):370-3.

9. Coffelt DW, Cobb CM, MacNeill S, Rapley JW, Killoy WJ. Determination of energy density threshold for laser ablation of bacteria An in vitro study. J Clin Periodontol. 1997;24(1):1-7.

10. Low SB, Mott A. Laser technology to manage periodontal disease: a valid concept? J Evid Based Dent Pract. 2014;14:154-9.

11. Convissar RA. Principles and practice of laser dentistry: Elsevier Health Sciences; 2015.

12. Finkbeiner RL. The results of 1328 periodontal pockets treated with the argon laser: Selective pocket thermolysis. J Clin Laser Med Surg. 1995;13(4):273-81.

13. Moritz A, Schoop U, Goharkhay K, Schauer P, Doertbudak O, Wernisch J, et al. Treatment of periodontal pockets with a diode laser. Lasers Surg Med. 1998;22(5):302-11.

14. Kreisler M, Al Haj H, d'Hoedt B. Clinical efficacy of semiconductor laser application as an adjunct to conventional scaling and root planing. Lasers Surg Med. 2005;37(5):350-5.

15. Üstün K, Erciyas K, Sezer U, Şenyurt SZ, Gündoğar H, Üstün Ö, et al. Clinical and biochemical effects of $810 \mathrm{~nm}$ diode laser as an 
adjunct to periodontal therapy: a randomized split-mouth clinical trial. Photomed Laser Surg. 2014;32(2):61-6.

16. Alves VTE, de Andrade AKP, Toaliar JM, Conde MC, Zezell DM, Cai S, et al. Clinical and microbiological evaluation of high intensity diode laser adjutant to non-surgical periodontal treatment: a 6-month clinical trial. Clin Oral Investig. 2013;17(1):87-95.

17. Moritz A, Gutknecht N, Doertbudak O, Goharkhay K, Schoop U, Schauer P, et al. Bacterial reduction in periodontal pockets through irradiation with a diode laser: a pilot study. J Clin Laser Med Surg. 1997;15(1):33-7.

18. De Micheli G, de Andrade AKP, Alves VTE, Seto M, Pannuti CM, Cai S. Efficacy of high intensity diode laser as an adjunct to non-surgical periodontal treatment: a randomized controlled trial. Lasers Med Sci. 2011;26(1):43-8.

19. Jose KA, Ambooken M, Mathew JJ, Issac AV, Kunju AP, Parameshwaran RA. Management of Chronic Periodontitis Using Chlorhexidine Chip and Diode Laser-A Clinical Study. J Clin Diagn Res. 2016;10(4):ZC76.

20. Zare D, Haerian A, Molla R, Vaziri F. Evaluation of the effects of diode $(980 \mathrm{~nm})$ laser on gingival inflammation after nonsurgical periodontal therapy. J Lasers Med Sci. 2014;5(1):27.

21. Dukić W, Bago I, Aurer A, Roguljić M. Clinical effectiveness of diode laser therapy as an adjunct to non-surgical periodontal treatment: a randomized clinical study. J. Periodontol. 2013;84(8):1111-7.

22. Borrajo JL, Varela LG, Castro GL, Rodriguez-Nunez I, Torreira MG. Diode laser (980 $\mathrm{nm})$ as adjunct to scaling and root planing. Photomed Laser Surg. 2004;22(6):509-12.

23. Saglam M, Kantarci A, Dundar N, Hakki SS. Clinical and biochemical effects of diode laser as an adjunct to nonsurgical treatment of chronic periodontitis: a randomized, controlled clinical trial. Lasers Med Sci. 2014;29(1):37-46.

24. Nguyen N-T, Byarlay MR, Reinhardt RA, Marx DB, Meinberg TA, Kaldahl WB. Adjunctive non-surgical therapy of inflamed periodontal pockets during maintenance therapy using diode laser: a randomized clinical trial. J. Periodontol. 2015;86(10):1133-40.

25. Marsh PD, Moter A, Devine DA. Dental plaque biofilms: communities, conflict and con- trol. Periodontol 2000. 2011;55(1):16-35.

26. Kamma J, Filippopoulou T, Vasdekis V. 980 $\mathrm{nm}$ diode laser adjunctive to non surgical periodontal treatment of chronic periodontitis: evaluation of clinical parameters. J Clin Periodontol. 2009;36:17-8. 